\title{
Zwei Autoren - ein Tisch - eine Sekretärin
}

\author{
Kooperatives Schreiben bei Negt und Kluge
}

\author{
Annegret Pelz, Christian Wimplinger
}

Oskar Negt und Alexander Kluge schreiben seit über $5^{\circ}$ Jahren gemeinsam Texte. ${ }^{1}$ Diese für beide alltägliche Schreibpraxis war seit den $1980 e r$ Jahren vielfach Gegenstand von Gesprächen und Interviews, ${ }^{2}$ weckt aber erst in jüngster Zeit das Interesse der Forschung. So stellt das Alexander-Kluge-Jahrbuch zum Stichwort: Kooperation (2017) die Zusammenarbeit des Sozialphilosophen Negt mit dem Autor, Filmemacher und Fernsehproduzenten Kluge in die Schreibtradition von Adorno und Horkheimer. ${ }^{3}$ Negt und Kluge selber führen ihre Kooperation auf die „Anfänge[] der studentischen Protestbewegung “4

1 Kluge und Negt begegneten einander 1968 während der studentischen Protestbewegung in Frankfurt, zwei Jahre später bietet Oskar Negt dem bereits als Filmemacher und literarischer Autor bekannten, jüngeren Kluge die Zusammenarbeit bezüglich eines Buches über Medien an. Das Ergebnis dieses Anstoßes ist der Band Öffentlichkeit und Erfahrung, der 1972 erscheint. Seither haben sie mit Geschichte und Eigensinn (1981) und Maßverhältnisse des Politischen (1992) im Zehnjahresrhythmus gemeinsam insgesamt drei umfangreiche Bücher geschrieben. Alle drei Bücher befassen sich mit politischer Ökonomie und Öffentlichkeit aus der Perspektive ihrer „subjektiven Mitgift der Menschen“ (Oskar Negt und Alexander Kluge, Der unterschätzte Mensch. Gemeinsame Philosophie in zwei Bänden, Frankfurt/Main: Zweitausendeins 2001, Bd. 1, S. 17).

2 John F. Hartle im Gespräch mit Oskar Negt, „Kooperationszusammenhänge kritischer Theorie“, in: Zeitschrift für kritische Theorie 46/47 (2018), S. 145-165; Oskar Negt, Rainer Stollmann und Christian Schulte, „Der Maulwurf kennt kein System. Oskar Negt im Gespräch mit Rainer Stollmann und Christian Schulte“, in: Rainer Stollmann und Christian Schulte (Hrsg.), Der Maulwurf kennt kein System. Beiträge zur gemeinsamen Philosophie von Oskar Negt und Alexander Kluge, Bielefeld: transcript 2005, S. 11-41; Alexander Kluge und Oskar Negt, „Öffentlichkeit als wertvolles Gut und die Idee der Gegenöffentlichkeit. Alexander Kluge und Oskar Negt im Gespräch“, in: Hans-Peter Burmeister (Hrsg.), Maßverhältnisse des Politischen. Öffentlichkeit und Erfahrung an der Schwelle zum 21. Jahrhundert, Rehburg, Loccum: Evangelische Akademie Loccum 2003, S. 37-55; Alexander Kluge und Oskar Negt, „Die Geschichte der lebendigen Arbeitskraft. Diskussion mit Oskar Negt und Alexander Kluge“, in: Dieter Hoffmann-Axthelm und Werner Siebel (Hrsg.), Technik und Sozialisation, Berlin: Ästhetik und Kommunikation 1982, S. 79-109.

3 Barbara Potthast, „,Dass der andere nichts denkt, was feindselig wäre - Kooperatives Denken bei Alexander Kluge“, in: Richard Langston et al. (Hrsg.), Stichwort: Kooperation. Keiner ist alleine schlau genug, Göttingen: V\&R unipress 2017, S. 103-113, hier S. 106.

4 Alexander Kluge, „Momentaufnahmen aus unserer Zusammenarbeit“, in: Wolfgang Lenk und Oskar Negt (Hrsg.), Kritische Theorie und politischer Eingriff: Oskar Negt zum 65. Geburtstag, Hannover: Offizin 1999, S. 25-41. Der Text ist in der Gesamtausgabe der gemeinsamen 
und die „Tage der Politischen Universität (28.-30. Mai 1968)“5 in Frankfurt zurück. Im Anschluss an die dort gemachten politischen Erfahrungen haben sie sich maßgeblich mit Öffentlichkeit, Arbeit, Enteignung in kapitalistischen Produktionsverhältnissen und - in Geschichte und Eigensinn (1981) - mit dem Begriff der Kooperation befasst. ${ }^{6} \mathrm{Zu}$ den Bedingungen ihres gemeinsamen Schreibens haben sie sich jedoch erst spät und - wie auch die kooperativen Verfasser Adorno/Horkheimer und Hardt/Negri ${ }^{7}$ - aus großer zeitlicher Distanz geäußert. In einem kurzen, Kluges 70. Geburtstag gewidmeten Text, der im Februar 2002 in der Frankfurter Rundschau erschienen ist, gibt Oskar Negt „Einblick in die Werkstatt“ und bemerkt: „Dass wir unseren Kooperationsprozess je öffentlich vorgestellt hätten, daran kann ich mich nicht erinnern“, doch „darf man sich“ die Frage, wie die dicken Bände gemeinsamer Arbeit zu Stande gekommen seien, „in unserem Alter wohl stellen.“8 In einer Festschrift zu Negts 65. Geburtstag macht Kluge in seiner Laudatio „Momentaufnahmen aus unserer Zusammenarbeit" (1999) außerdem auf ein Darstellungsproblem aufmerksam:

Es ist eine „Tatsache“ und es kann als „bekannt“ gelten, daß wir jetzt seit 28 Jahren zusammen arbeiten. Es ist aber nicht einfach, dieses Bekanntsein und die Tatsache für sich selbst abzubilden. Man muss die konkreten Tage, die wir zusammen verbracht haben, erst wieder einsammeln. Ich bemerke, während ich Momentaufnahmen einzusammeln versuche (und einsammeln heißt auf griechisch „lesen“), also in unserer Lebenszeit lese, daß zunächst Einzelbilder im Vordergund stehen[.] ${ }^{9}$

Was hier „rasch und als Bilder in Erscheinung “10 tritt und sich einsammeln und zusammenlesen lässt, sind einzelne, aus der chronologischen Ordnung

Philosophie Negt und Kluges, Der unterschätzte Mensch, mit leichten Veränderungen erneut abgedruckt, vgl. Negt/Kluge, Der unterschätzte Mensch (Anm. 1), Bd. 1, S. 5-19. Im Folgenden wird aus der Ausgabe Der unterschätzte Mensch zitiert, hier S. 6. Kluge, „Momentaufnahmen“ (Anm. 4), S. 12.

6 Vgl. Oskar Negt und Alexander Kluge, Geschichte und Eigensinn (1981), in: Negt/Kluge, Der unterschätzte Mensch (Anm. 1), Bd. 2, S. 183-193.

7 Vgl. Theodor W. Adorno, „Offener Brief an Max Horkheimer“ (1965), in: ders., Vermischte Schriften I. Theorien und Theoretiker. Gesellschaft, Unterricht, Politik, hrsg. von Rolf Tiedemann unter Mitwirkung von Gretel Adorno, Susan Buck-Morss und Klaus Schultz, Frankfurt/Main: Suhrkamp 2003, S. 153-163; Michael Hardt, „How to write with four hands", in: Genre 46/2 (2013), S. 175-182.

8 Oskar Negt, „Meister der Verschlüsselungen. Einblicke in die Werkstatt: Zum 7o. Geburtstag von Alexander Kluge, Frankfurter Rundschau vom 6. Februar 2002", in: Burmeister (Hrsg.), Maßverhältnisse des Politischen (Anm. 2), S. 193-196, hier S. 193.

9 Kluge, „Momentaufnahmen“ (Anm. 4), S. 5.

10 Ebd. 
herausgebrochene Momentaufnahmen von gegenseitigen Besuchen und Ritualen am gemeinsamen Arbeitsplatz, nicht aber vom Arbeitsprozess selbst. Dessen „konzentrierte[] Augenblicke“ sind, wie der Text betont, in die Bücher eingegangen, in der Erinnerung jedoch weitgehend verschwunden. ${ }^{11}$ Die Zusammenarbeit, aus der zwei dicke Bände "gemeinsamer Philosophie“ hervorgegangen sind, bezeugt jedoch ein Foto, das Kluge im Text erwähnt und das die beiden „unter einer gemeinsamen Lampe, am gemeinsamen Arbeitstisch $^{\text {"12 }}$ einander gegenübersitzend und über Bücherberge hinweg diskutierend zeigt (Abb. 7.1).

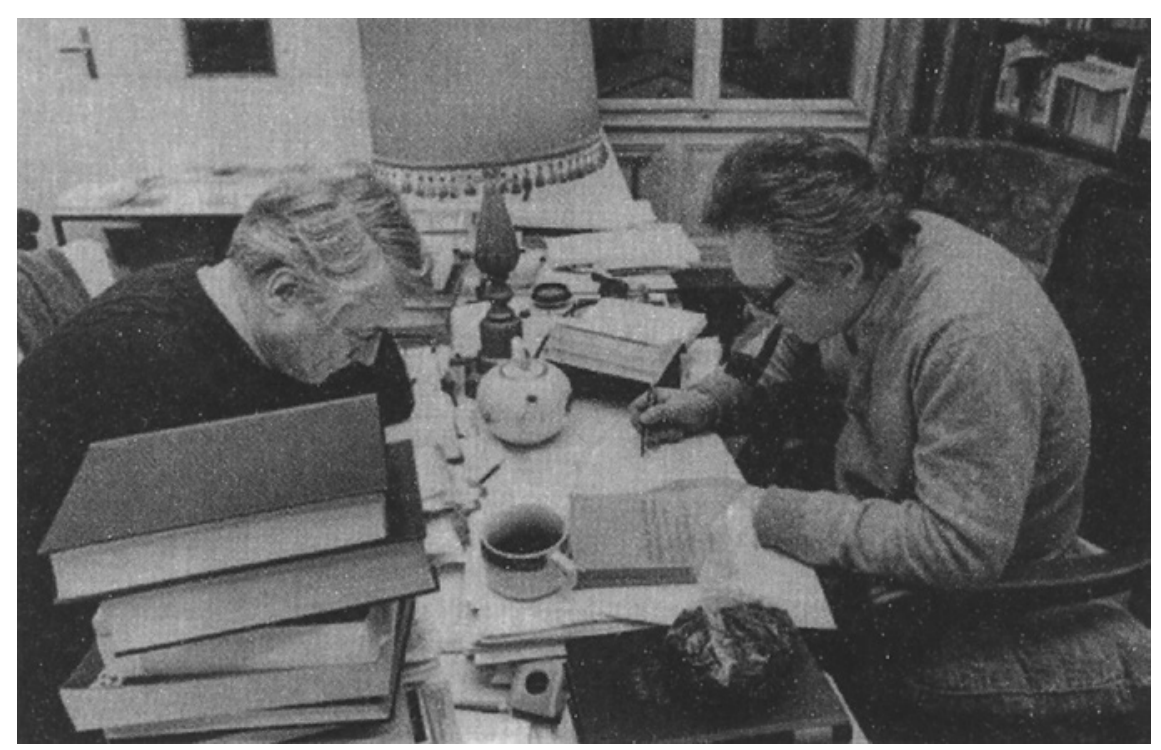

Abb. 7.1 Oskar Negt und Alexander Kluge am gemeinsamen Arbeitstisch.

Kluge, der in seinem Erinnerungsversuch auch auf Abbildungsschwierigkeiten stößt, wird von dieser Aufnahme nicht erwarten, dass sie das gemeinsame Schreiben veranschaulicht. $\mathrm{Zu}$ entschieden haben schon Karl Kraus und

11 Ebd. Dass die Augenblicke in der Erinnerung verschwunden sind, ist nach Marx' Erinnerungstheorie, auf die sich auch Negt und Kluge beziehen, ein Anzeichen für einen gelungenen Produktionsprozess: „Im gelungenen Produkt wird die Erinnerung an den Prozeß [seiner Herstellung, Anm.] aufgezehrt, während erst die zerbrochene Gabel den Menschen darauf bringt, darüber nachzudenken und sich daran zu erinnern, ob etwa im Produktionsprozeß selber etwas schief gelaufen ist" (Negt/Kluge, Geschichte und Eigensinn [Anm. 6], S. 105).

12 Kluge, „Momentaufnahmen“ (Anm. 4), S. 6. 
Ingeborg Bachmann darauf hingewiesen, dass ein Foto, das Schriftsteller in ihrer Werkstatt abbildet, ein pseudonatürliches Schauspiel der Textproduktion bietet. ${ }^{13}$ Wo sich die Darstellung der Zusammenarbeit als Problem erweist, verweisen Negt und Kluge jedoch stets auf den ins Bild gesetzten Arbeitstisch als Bedingung für das Zustandekommen eines gemeinsamen Werkes. Auf eine Nachfrage von Jürgen Habermas, was denn „,Wort für Wort gemeinsam geschrieben' konkret bedeute“, beschreiben sie die Situation, indem sie auf den Tisch zeigen. ${ }^{14}$ Dieser ermögliche eine „Art des Diskussionsund Arbeitsprozesses" und die Ausprägung eines Verhaltens, das so etwas wie "kommunikative[s] Urvertrauen“ und die Haltung eines „absichtslosen Sich-der-Diskussion-Anvertrauen[s]" zur Voraussetzung habe. ${ }^{15}$ Eine kritische Haltung, ${ }^{16}$ die Kluge wesentlich mit der Integrität Oskar Negts verbindet. Gemeint ist das

Vertrauen darauf, daß wir uns nur hinzusetzen brauchen, und es wird gelingen, gemeinsam problematische Fragen nebeneinanderzustellen, sie nacheinander $\mathrm{zu}$ analysieren, unmerklich zu einer Einigung zu kommen, wie man sich zu diesen Fragen entscheidet und sie darstellt. ${ }^{17}$

Das gemeinsame Sitzen am Tisch ermöglicht eine Arbeitsform, die Kluge "gefügeartige Arbeit" nennt. Zwei Geschichten mit dem Titel Gefügeartige Arbeit (2003, 2014) beschreiben diese als eine "Spitzenleistung [...] in der Evolution der Arbeitsvermögen“,18 die dem „Sich-aufeinander-Einlassen“ selbstbewusster Fachkräfte entspricht. ${ }^{19}$ Historische Vorbilder gefügeartiger

13 Vgl. Ingeborg Bachmann, „[Rede zur Verleihung des Anton-Wildgans-Preises]“, in: dies., Werke, hrsg. von Christine Koschel, Inge von Weidenbaum und Clemens Münster, 4 Bde., München, Zürich: Piper 41993, Bd. 4, S. 294-297, hier S. 294f.; Karl Kraus, „In der Werkstatt", in: Die Fackel 14, Nr. 347/348 (27.04.1912), S. 49.

14 Oskar Negt, „Über Vertrauen und Kooperation“, in: Der Deutschunterricht 3 (2012), S. 18-24, hier S. 19 .

15 Kluge, „Momentaufnahmen“ (Anm. 4), S. 5 f.

16 Diese Haltung entspricht der „inneren Struktur des politischen Protestes und der Kritischen Theorie“ (Kluge, „Momentaufnahmen“ [Anm. 4], S. 6), weil sie die zumeist ausgeklammerte Lebenserfahrung in die Protest- und Textarbeit integriert. Die persönlichen, oft im Privaten verborgenen und in öffentlichen Diskussionen ausgegrenzten Erfahrungen sind der entscheidende Ausgangspunkt kritischer Diskussionen in der studentischen Protestbewegung.

17 Kluge, „Momentaufnahmen“ (Anm. 4), S. 5.

18 Alexander Kluge, „Gefügeartige Arbeit“, in: ders., Das fünfte Buch. Neue Lebensläufe. 402 Geschichten, Berlin: Suhrkamp 2012, S. 164; Alexander Kluge, „Gefügeartige Arbeit“, in: ders., Die Lücke, die der Teufel lässt. Im Umfeld des neuen Jahrhunderts, Frankfurt/Main: Suhrkamp 2005, S. 721-723.

19 Kluge, „Momentaufnahmen“ (Anm. 4), S. 6. 
Kooperationsformen sieht Kluge zudem im sokratischen Dialog und in der Gelehrtenrepublik der Aufklärung, wobei er an diesen beiden historischen Konstellationen bemängelt, dass sie die privaten Lebenszusammenhänge der Gesprächspartner ausgrenzen. Erst deren Einbeziehung macht gefügeartige Arbeit zu etwas Lebendigem, Gemeinschaftlichem und letztlich auch zu etwas Artistischem. ${ }^{20}$

\section{Gefügeartige Arbeit - konstellatives Erzählen}

Dass Negt und Kluge zusammenarbeiten und ihre Texte gemeinschaftlich verfassen, ist vielfach belegt. Beschrieben wird die Zusammenarbeit als "kooperatives Denken“, „verteilte Autorschaft“ oder auch „Zweistimmigkeit“. ${ }^{21}$ Unsere Frage, inwiefern die kooperative Textproduktion auch als kollektives Schreiben bezeichnet werden kann, setzt dort ein, wo der Begriff der Momentaufnahme nicht nur Kluges Erinnerungsversuch, sondern auch Negt und Kluges „KONSTELLATIVE ERZÄHLWEISE“22 benennt. Hier erlaubt das „Prinzip des CROSS-MAPPING, die Anwendung einander widersprechender Kartierungen, Methoden oder Theorien“, eine polyperspektivische, multimediale und a-lineare Darstellung der Transformationen „äußerliche[r] Formen der Arbeit“ ins „Innere[] der Menschen“. ${ }^{23}$ So sollen die in konstellativer Erzählweise verschriftlichten Momente geschichtlicher Erfahrung augenblickshafte Einblicke in ein kollektives Unbewusstes gewähren, ohne auf kritische Überprüfung zu verzichten. Über die kritische Reflexion hinaus erheben Negt und Kluge den Anspruch, die theoretische Arbeit unmittelbar in praktische Aktion zu überführen. Sie sehen diese Art von „Intelligenzarbeit“ (im Unterschied zur großen Systemphilosophie, die sich auf vergangene Realitäten bezieht ${ }^{24}$ ) als Tätigkeit von „Einsichtlern', Erkennende[n], Sortierer[n], Sammler[n], Boten, Experimentatoren, Erfinder[n], Bücherfreunde[n], Unterhalter[n], Baumeister[n], Konstrukteure[n]“..25 Das mit diesen Finde- und

20 Ebd.

21 Potthast, „Dass der andere nichts denkt“ (Anm. 3), S. 133-139; Hans-Peter Burmeister, „Zwei Stimmen aus Deutschland“, in: Schulte/Stollmann (Hrsg.), Der Maulwurf(Anm. 2), S. 83-92, hier S. 83 .

22 Kluge, Das fünfte Buch (Anm. 18), S. 177. Birgit Erdle und Annegret Pelz (Hrsg.), Augenblicksaufzeichnung - Momentaufnahme. Kleinste Zeiteinheit, Denkfigur, mediale Praktiken, Paderborn, Fink/Brill 2020 und darin: Christian Wimplinger, „Der merkwürdige Moment. Jetztzeit bei Alexander Kluge“, S. 137-153.

23 Ebd., S. 176.

24 Von dieser grenzen sich Negt/Kluge, Geschichte und Eigensinn (Anm. 6), S. 84, ab.

25 Negt/Kluge, Geschichte und Eigensinn (Anm. 6), S. 369. 
Konstruktionstätigkeiten verbundene Textverfahren der Momentaufnahme ist insofern konstellativ, als die Zitate zunächst mündlich gesammelt, Texte angeordnet und historische und theoretische Passagen nebeneinandergestellt werden. Auf die in dieser konstellativen Praxis entstehenden Beziehungen kommt es an. In „Momentaufnahmen aus unserer Zusammenarbeit“ heißt es:

Momentaufnahmen dieser Art werden in Notzeiten, zu Beginn von Revolutionen und im solidarischen Kontext beschrieben. Es geht um das Verhalten fusionierender Gruppen, um etwas, das nicht festgelegt ist und sich auch nicht festlegt. ${ }^{26}$

Die im Zitat verwendete Bezeichnung „fusionierende Gruppe“ geht auf Sartre zurück. Kluge greift sie in der Geschichte Was ist eine, fusionierende Gruppe?? Rosa Luxemburg und die Revolution von 1905 (2003) auf. ${ }^{27}$ In Kluges literarischer Bearbeitung, die historische Begebenheiten frei verhandelt, ${ }^{28}$ reist Rosa Luxemburg 1905 nach Kiew, um die Erfahrungen der Aufständischen über den „im Augenblick der Umwälzung“29 entstehenden „REVOLUTIONÄREN GESAMTARBEITER “30 in Form von Augenzeugen-Berichten einzusammeln. Kluge zufolge wirkt die Revolutionsbewegung anfänglich inkludierend: Ein Taschendieb „vergaß sein Geschäft", ein Anwalt die Gesetzeswidrigkeit der Zusammenrottung. ${ }^{31}$ Kluge beschreibt, wie Rosa Luxemburg Möglichkeiten sondiert, das temporär limitierte "RIESENBABY REVOLUTION“ in eine stabile und fortdauernde „NEUE GESELLSCHAFT“ zu überführen, ohne dafür aber taugliche Mittel auszumachen. ${ }^{32}$ Ihre Augenzeugen-Berichte in Form von Momentaufnahmen bezeugen intensive Erfahrungen von Gemeinschaftsbildungen, wie sie in historisch offenen Augenblicken entstehen, bei gleichzeitiger Instabilität dieser Gemeinschaft.

Vor dem Hintergrund der instabilen und krisenhaften Nachkriegsgeschichte wollen auch die Publikationen von Negt und Kluge kritische Momente der deutschen Geschichte in Momentaufnahmen bezeugen. Sie mustern „die

26 Kluge, „Momentaufnahmen“ (Anm. 4), S. 10.

27 Alexander Kluge, „Momentaufnahmen der politischen Revolution“, in: ders., Tür an Tür mit einem anderen Leben. 350 neue Geschichten, Frankfurt/Main: Suhrkamp 2006, S. 36o373, hier S. 362f. Vgl. auch Jean-Paul Sartre, Kritik der dialektischen Vernunft 1 . Theorie der gesellschaftlichen Praxis (1960), Reinbek bei Hamburg: Rowohlt 1967, S. 383-385.

28 Vgl. Holger Politt, „Unter Blitz und Donner. Zusammenstoß zweier Zeitalter“, in: Rosa Luxemburg, Arbeiterrevolution 1905/o6. Polnische Texte, hrsg. von Holger Politt, Berlin: Dietz 2014, S. 9-34, hier S. 19f.

29 Kluge, „Momentaufnahmen der politischen Revolution“ (Anm. 27), S. 362.

30 Ebd., S. 363 .

31 Ebd., S. 362.

32 Ebd., S. 363 . 
Veränderungen und Tendenzen der zurückliegenden Dekade im Übergang zur nächsten [...] und [beurteilen diese] in Hinblick auf ihre liegengebliebenen und unabgegoltenen Reste. “33 So behandelt Öffentlichkeit und Erfahrung (1972) die Entstehungsmöglichkeiten einer proletarischen Öffentlichkeit, die in der studentischen Protestbewegung „in embryonaler Form“34 vorgebildet sind. Derartige Entwicklungspotentiale einer anderen Öffentlichkeit hat Öffentlichkeit und Erfahrung nicht nur zum Gegenstand, sondern war ihr integraler Bestandteil. Bereits vor Erscheinen zirkulierte das Buch im Merve-Kollektiv um Peter Gente ${ }^{35}$ und diente dem Verlag als Schlüssel bei der Konstitution einer, „zumindest begrifflich, der amerikanischen ,counterculture nachempfundenen“,36 proletarischen Gegenöffentlichkeit, in der aktuelle Theorie in Paperbacks schnell und erschwinglich zugänglich sein sollte.

Geschichte und Eigensinn (1981) wiederum erzählt von der Entwicklung lebendiger Arbeitsvermögen in Produktionsstätten, in Beziehungen, im Krieg, im Protest - und von der Enteignung dieser Vermögen im Kapitalismus sowie von ihrer Gegenwehr in Form von eigensinnigen „Feingriffen“ und terroristischen "Gewaltgriffen" im Horizont des Deutschen Herbstes. ${ }^{37}$ Das intentional „Fragment ${ }^{\text {"38 }}$ gebliebene Buch umfasst in der Erstausgabe 1.283 Seiten, die zwar einem plausiblen Aufbau folgen, eine Ökonomie der lebendigen Arbeitskraft skizzieren und diese insbesondere anhand der Geschichte Deutschlands vertiefen, und die dennoch, aufgrund der Heterogenität der zusammengetragenen Materialien, Texte, Formate und Bilder, oft mit einer „Materialcollage“ oder einem „Trümmerfeld“ verglichen werden. ${ }^{39}$

33 Wolfgang Bock, „Exemplarische Reflexionen einer Dekade. ,Maßverhältnisse des Politischen“', in: Schulte/Stollmann (Hrsg.), Der Maulwurf(Anm. 2), S. 107-130, hier S. 107. Zuvor bemerkt diese historische Konstellierung Rainer Stollmann, „Der unterschätzte Mensch. Der Text als anschlussfähiges Netzwerk“, in: Burmeister (Hrsg.), Maßverhältnisse des Politischen (Anm. 2), S. 13-24, hier S. 17.

34 Oskar Negt und Alexander Kluge, „Öffentlichkeit und Erfahrung. Zur Organisationsanalyse von bürgerlicher und proletarischer Öffentlichkeit", in: dies., Der unterschätzte Mensch (Anm. 4), Bd. 1, S. 362-674, hier S. 441.

35 Vgl. Philipp Felsch, Der lange Sommer der Theorie. Geschichte einer Revolte. 1960-199o, München: Beck 2015, S. 89 .

36 Ebd.

37 Zur Unterscheidung von Fein- und Gewaltgriffen siehe Negt/Kluge, Geschichte und Eigen$\operatorname{sinn}$ (Anm. 6), hier S. 20-22.

38 Ebd., S. 1245 .

39 Christian Schulte, „Cross-Mapping. Aspekte des Komischen. ,Mit der Straßenkarte von Groß-London den Harz durchwandern“', in: Schulte/Stollmann (Hrsg.), Der Maulwurf (Anm. 2), S. 219-232, hier S. 219f. Die Beschreibung von Kluges Texten als ,Trümmerfeld“ nimmt Schulte aus: Hans Magnus Enzensberger, „,Ein herzloser Schriftsteller'“, in: Der Spiegel 1 (1978), S. 81-83, hier S. 81 . 
Während für Öffentlichkeit und Erfahrung und für Geschichte und Eigensinn gilt, dass die Bücher "Wort für Wort gemeinsam hergestellt" ${ }^{40}$ wurden, besteht das dritte gemeinsam verfasste Buch, Maßverhältnisse des Politischen (1992), zu großen Teilen aus kompiliertem Material bereits separat und in anderen Kontexten publizierter Texte. ${ }^{41}$ Diese überprüfen die subjektiven Gebrauchswerte realpolitischer Vorgänge, etwa der deutschen Wiedervereinigung, für die Bildung eines stabilen Gemeinwesens, ${ }^{42}$ indem einzelne, ins Gedächtnis tretende Ereignisse zusammengestellt werden. Das Besondere ist hier, dass diese Textsammlungen im Laufe der Jahre immer wieder erweitert und in neuen Zusammenstellungen und Übersetzungen publiziert werden, ${ }^{43}$ so dass ein bewegliches Archiv mit historischen Momentaufnahmen entsteht, in dem Erfahrung aufbewahrt, überprüft, neu zusammengelesen und revidiert werden kann. ${ }^{44}$

\section{Kollektive und kooperative Praxis im erweiterten Kontext der Studentenbewegung}

Die Kooperation von Negt und Kluge wurzelt, wie auch diejenige von Deleuze und Guattari, in der studentischen Protestbewegung. Dies- und jenseits des Rheins wurden Autoritäten kritisiert und versucht, das unterdrückte Subjekt der Geschichte zu bestimmen: ein Subjekt, das seine Repression durch das kapitalistische System zwar nicht (mehr) bewusst wahrnimmt, für eine gesamtgesellschaftliche Umwälzung aber die treibende revolutionäre Kraft sein muss. ${ }^{45}$ In diesem revolutionären Prozess suchten die Intellektuellen

\footnotetext{
40 Kluge, „Momentaufnahmen“ (Anm. 4), S. 9.

41 Oskar Negt und Alexander Kluge, Maßverhältnisse des Politischen. 15 Vorschläge zum Unterscheidungsvermögen, Frankfurt/Main: Fischer 1992.

42 Vgl. ebd., insbes. S. 303-309.

43 Ins Englische sind übersetzt: Oskar Negt und Alexander Kluge, Public Sphere and Experience. Toward an Analysis of the Bourgeois and Proletarian Public Sphere, foreword by Miriam Hansen, translated by Peter Labanyi, Jamie Owen Daniel and Assenka Oksiloff, Minneapolis, London: University of Minnesota Press 1993; Alexander Kluge und Oskar Negt, History and Obstinacy, translated by Richard Langston with Cyrus Shahan, Martin Brady, Helen Hughes and Joel Golb, New York: Zone Books 2014.

44 Der letzte Wort für Wort gemeinsam verfasste Text der beiden Autoren ist: Oskar Negt und Alexander Kluge, „Love Politics. Eigensinn der Intimität. Essay“, in: Alexander Kluge, Das Labyrinth der zärtlichen Kraft. 166 Liebesgeschichten, Frankfurt/Main: Suhrkamp 2009, S. 519-568.

45 Beschrieben unter anderem bei Dick Howard, „Telos. Wanderwege der Neuen Linken“, in: Zeitschrift für Ideengeschichte XI/3 (2017), S. 5-21, hier S. 12f. Zu dem um die Mitte des 20. Jahrhunderts ausgerufenen, so genannten ,collaborative turn' und der Suche
} 
die Verbindung zur Arbeiterschaft sowie nach neuen Kollektiv- und „Gemeinschaftsformen [...] außerhalb herrschender Politik und Zwangsmittel“. ${ }^{46}$ Zentral für die Kollektiv-Idee dieser Zeit war die Frage, wie sich Gemeinschaften unabhängig von äußeren Sachlagen selbst gesetzgebend konstituieren können. Ausgehend von seiner psychiatrischen Arbeit bestimmt Félix Guattari solche unabhängigen Gruppen als Subjektgruppen (groupes sujets), die im Gegensatz zu den unterworfenen Gruppen (groupes assujets) „sich aus einem inneren Gesetz heraus “47 selbst bestimmen. ${ }^{48}$ Jean Paul Sartre stellt in der Kritik der dialektischen Vernunft (196o, dt. 1967) eine, so der Untertitel, Theorie der gesellschaftlichen Praxis und eine Theorie zur Entstehung von Sozialgebilden auf. Diese setzt fusionierende Gruppen als Keimzelle voraus und zeigt „den Übergang der unterdrückten Klassen aus dem Kollektivzustand zur revolutionären Gruppenpraxis" auf. ${ }^{49}$ In Deutschland spielte in diesem Zusammenhang Marx' Begriff des Gesamtarbeiters und die Überlegung, wie man „relevante[] Teile wissenschaftlicher Intelligenz“ ${ }^{50}$ in denselben integrieren könnte, eine wichtige Rolle. ${ }^{51}$

nach kollektiven Handlungsformen jenseits etablierter Institutionen in der Kunstwelt, siehe Sabeth Buchmann und Tom Holert, „Materielle Praxis, Wissensproduktion. Kollektivität und Kollaborativität als Fluchtlinien des Künstlerischen“, in: ,Mit-Sein: Gemeinschaft - ontologische und politische Perspektivierungen, hrsg. von Elke Bippus, Jörg Huber und Dorothee Richter, Wien, New York: Springer/Zürich: Edition Voldemeer 2010, S. $189-213$.

46 Bernd Rabehl, „Zur archaischen Inszenierung linksradikaler Politik. Ursache und Auswirkungen des politischen Existentialismus in der Studentenrevolte 1967/68“, in:Wolfgang Kraushaar (Hrsg.): Frankfurter Schule und Studentenbewegung. Von der Flaschenpost zum Molotowcocktail 1946-1995, Hamburg: Rogner \& Bernhard 1998, S. 34-64, hier S. 58.

47 Gilles Deleuze und Félix Guattari, Kapitalismus und Schizophrenie 1. Anti-Ödipus (1972), aus dem Französischen übersetzt von Bernd Schwibs, Frankfurt/Main: Suhrkamp 1977, S. 362 .

48 Die Problematik kommt aus der psychiatrischen Arbeit von Félix Guattari, „Einführung in die institutionelle Psychotherapie“, in: ders., Psychotherapie, Politik und die Aufgaben der institutionellen Analyse, mit einem Vorwort von Gilles Deleuze, aus dem Französischen übersetzt von Grete Osterwald, Frankfurt/Main: Suhrkamp 1976, S. 82-97, hier S. 85.

49 Sartre, Kritik der dialektischen Vernunft (Anm. 27), S. 375f.

50 Hans-Jürgen Krahl, „Thesen zum allgemeinen Verhältnis von wissenschaftlicher Intelligenz und proletarischem Bewusstsein" (1969), in: ders., Konstitution und Klassenkampf. Zur historischen Dialektik von bürgerlicher Emanzipation und proletarischer Revolution. Schriften, Reden und Entwürfe aus den Jahren 1966-1970, 5. Aufl., Frankfurt/ Main: Neue Kritik 2008, S. 336-353, hier S. 347.

$51 \quad$ Negt und Kluge sehen das Problem kaum erfolgter Kollektivbildung zwischen Kopf- und Handarbeitenden darin, dass die ersteren in einer handwerklichen Produktionsweise verharren, während sich die letzteren industrialisieren. Vgl. Negt/Kluge, Geschichte und Eigensinn (Anm. 6), S. 442f. 
Wie kollektive Erfahrung öffentlich organisiert werden kann, beschäftigt Negt und Kluge in vielfältigen institutionellen und beruflichen Kooperationen: Kluge ist in Frankfurt juristischer Berater am Institut für Sozialforschung, Negt wissenschaftlicher Assistent von Habermas und Repräsentant des SDS. Außerdem ist Kluge Leiter des Ulmer Instituts für Filmgestaltung. Hinzu kommen filmische Koproduktionen mit Rainer Werner Fassbinder, Edgar Reitz, Wim Wenders, Werner Herzog, Volker Schlöndorff und Hans-Jürgen Syberberg, die in den 1970er Jahren im Begriff des Kollektivfilms die Idee des Kollektivs aufgreifen, ebenfalls in gefügeartiger Form..$^{52}$ Die Kooperationen setzen sich fort in der Zusammenarbeit mit Aust, Schlöndorff und Engstfeld und münden Ende der 1980er Jahre in Spiegel TV und in DCTP, Developing Company for Television Program, einer Produktionsfirma für Fernsehprogramme unter Kluges Beteiligung. Parallel zu diesen beruflichen, filmischen und künstlerischen Netzwerken laufen die Kooperationen auf der Ebene der Buchproduktion. Kluge arbeitet mit dem Buchgestalter bei Zweitausendeins, Franz Greno, ${ }^{53}$ publiziert mit Reinhard Jirgl, ${ }^{54}$ Ferdinand von Schirach, ${ }^{55}$ Joseph Vog ${ }^{56}$ sowie Ben Lerner ${ }^{57}$ und veröffentlicht gemeinsam mit dem Fotografen Stefan Moses das Buch Le Moment fugitif, in dem die Flüchtigkeit in der Produktion von Bildern und Texten ihrer absichtsvollen Planbarkeit gegenübergestellt wird. ${ }^{58}$ Aus Kooperationen mit bildenden Künstlern gehen Katalogpublikationen,

52 Als Beispiel gilt Deutschland im Herbst (1978), ein Episodenfilm, zu dem jeder Regisseur eine eigene Episode beisteuert.

53 „Ende der siebziger und in den achtziger Jahren ergibt sich durch die Verwendung von Bildmaterialien bei den Buchpublikationen bei Zweitausendeins, also Geschichte und Eigensinn (1981), Die Patriotin (1979) und Die Macht der Gefühle (1984) ein Austausch mit dem Buchgestalter Franz Greno" (Rainer Stollmann, Thomas Combrink und Gunther Martens: „Editorial“, in: Langston et al. (Hrsg.), Stichwort [Anm. 3], S. 11-17, hier S. 129).

54 Vgl. Alexander Kluge, Das Bohren harter Bretter, mit einem Gastbeitrag von Reinhard Jirgl, Berlin: Suhrkamp 2011; sowie Alexander Kluge, 30. April 1945. Der Tag, an dem Hitler sich erschoß und die Westbindung der Deutschen begann, mit einem Gastbeitrag von Reinhard Jirgl, Berlin: Suhrkamp 2014.

55 Vgl. Alexander Kluge und Ferdinand von Schirach, Die Herzlichkeit der Vernunft, München: Luchterhand 2017.

56 Vgl. Alexander Kluge und Joseph Vogl, Senkblei der Geschichten. Gespräche, Zürich: diaphanes 2020; Alexander Kluge und Joseph Vogl, Soll und Haben. Fernsehgespräche, Zürich: diaphanes 2008.

57 Vgl. Alexander Kluge und Ben Lerner, Schnee über Venedig, Leipzig: Spector Books 2018.

$5^{8}$ Vgl. Alexander Kluge und Stefan Moses, Le moment fugitif, Wäderswil: Nimbus 2014. 
Filmeditionen und Ausstellungen mit Anselm Kiefer, ${ }^{59}$ Georg Baselitz, ${ }^{60}$ Gerhard Richter ${ }^{61}$ sowie Thomas Demand und Anna Viebrock ${ }^{62}$ hervor. Grundsätzlich gilt für alle diese Gemeinschaftsprojekte Kluges Aussage über die Zusammenarbeit mit Reinhard Jirgl: „Erzählen ist gesellig. Bei der Arbeit bin ich ungern allein. ${ }^{63}$

\section{Tischszene}

Die Kooperationsform, auf die sich in der Protestbewegung eine "affirmative Hoffnung" richtet, hat Selbstregulierung zur Voraussetzung. ${ }^{64}$ Von fremdregulierenden Ordnungsformen („Kommandogewalt ${ }^{\star 65}$ ) unterscheiden sich selbstregulierende Abläufe dadurch, dass sie sich in einem Lebensbereich autonom herausbilden. Sie finden beim Menschen auf verschiedenen Ebenen statt: innerhalb der ersten Natur auf der Ebene von „Zellen, Haut, Körper, Hirn“, ${ }^{66}$ innerhalb der zweiten Natur in „Lieben, Wissen, Trauern, Erinnern, Familiensinn, Hunger nach Sinn, kollektiven Aufmerksamkeiten“, 67 oder z.B. „[i]n einer Wohngemeinschaft, [in der] zwei Mitglieder an einem Manuskript [arbeiten]“. ${ }^{68}$ Die teils sehr abstrakte Begriffsbildung, über die es weiter heißt,

59 Für den Katalog Anselm Kiefer, Die Ungeborenen, mit Texten von Alexander Kluge, hrsg. von Arne Ehmann, Paris: Galerie Thaddaeus Ropac 2012, schreibt Kluge Geschichten, fünf Jahre später ist die gemeinsame DVD Alexander Kluge und Anselm Kiefer, Der mit den Bildern tanzt. Filme und Dialoge, Berlin: Suhrkamp 2017, in der filmedition suhrkamp erschienen.

6o Vgl. Georg Baselitz und Alexander Kluge, Weltverändernder Zorn. Nachricht von den Gegenfüßlern, Berlin: Suhrkamp 2017. Im Jahr davor erschien der Ausstellungskatalog: Max Hollein und Eva Mongi-Vollmer (Hrsg.), Georg Baselitz - die Helden, München: Hirmer 2016, mit „15 Geschichten zu Bildern von Georg Baselitz aus den Jahren 1965 und 1966“ (S. 64-157).

61 Dies gilt für die Bücher Alexander Kluge und Gerhard Richter, Dezember. 39 Geschichten, 39 Bilder, Berlin: Suhrkamp 2010, und Alexander Kluge und Gerhard Richter, Nachricht von ruhigen Momenten. 89 Geschichten, 64 Bilder, Berlin: Suhrkamp 2013.

62 Vgl. Thomas Demand, Alexander Kluge und Anna Viebrock, The boat is leaking. The captain lied, Mailand: Fondazione Prada 2017.

63 Kluge, Das Bohren harter Bretter (Anm. 54), S. 7.

64 Vgl. Negt/Kluge, Geschichte und Eigensinn (Anm. 6), S. 70.

65 Ebd., S. 53 .

66 Ebd., S. 45.

67 Ebd.

68 Ebd., S. 54. 
„[d]ie Regulierung ist in Wirklichkeit eine Auseinandersetzung der lebendigen Arbeit mit sich selbst", thematisiert in Geschichte und Eigensinn auch den gemeinsamen Schreibprozess. ${ }^{69}$ Selbstregulierend sind etwa Begegnungen zwischen Dingen und Menschen, das heißt von "willenstarke[n] Leute[n]“ mit der „Tücke des Objekts“, z.B. mit eigenwilligen Möbeln im Stummfilm.70 Damit die Selbstregulierung funktioniert, bedarf es der „Anerkennung der Gesetze, nach denen Möbel [...] mit menschlichen Griffen kooperieren",71 einer Anerkennung, die Negt und Kluge auch „bewusste Zuarbeit" und „Übersetzung" nennen. ${ }^{72}$ So wie Freud während einer Behandlung „keinen Patienten mit einem Automaten" verwechsle, ${ }^{73}$ ist es Negt und Kluge zufolge wichtig, lebendige Menschen und tote Objekte zu unterscheiden. Sie begreifen diese nicht als Akteure im Latour'schen Sinn, beobachten aber selbstregulative Kreisläufe an ihrem Arbeitstisch, wenn dieser in einer überhitzten Diskussion zwischen Negt und Kluge eine steuernde Funktion einnimmt: „Sobald Nervosität auftrat, haben wir den gemeinsamen Tisch verlassen“, schreibt Negt in seinem Werkstattbericht. ${ }^{74}$ Nach einer freundlichen Verabschiedung, nach der Rückkehr an die Wohnorte gibt es Phasen, in denen der gemeinsame Tisch für Monate verlassen und die Produktion unterbrochen wird. Dann setzen sich die beiden zu einem neuen Termin „an denselben Tisch mit den liegen gebliebenen Manuskripten und Büchern" und nehmen die Arbeit an "genau [...] den Sätzen" wieder auf, bei denen sie gestoppt worden war. ${ }^{75}$ Der Tisch konserviert die strittige Situation, während die Autoren darauf vertrauen, dass die aufgetretene Nervosität sich in anderen Kontexten eigentätig rückbildet, um danach wieder „rasch [...] eine Situation herzustellen, die für Kooperation geeignet ist ${ }^{\prime} .76$ Als Konservator von strittigen Sachlagen und zur Selbstregulierung von Arbeit wird der Tisch unter der Rubrik „Zivilisationsprodukt, nicht Natur"77 subsumiert. Im Text wird er nicht als ein mit der Textproduktion unmittelbar verknüpftes Ding, d.h. als theoretisches Objekt der Schriftproduktion, reflektiert, sondern der Tisch bleibt ein zuhandenes Mittel. Werden Tischszenen im Text aufgerufen, dienen diese den Präliminarien der Einrichtung und der Beschreibung einer routinierten, kooperativen Arbeitsatmosphäre:

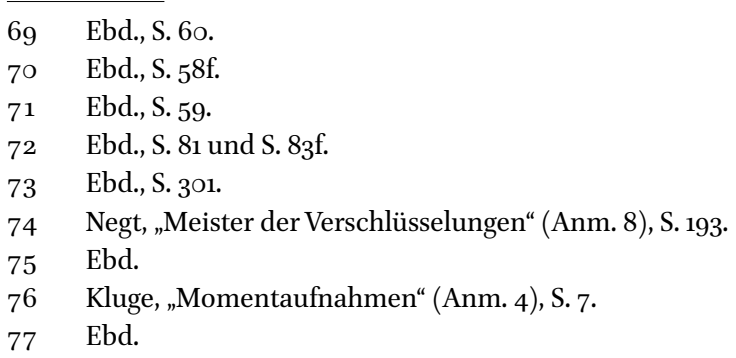


Wir essen im Wienerwald Ecke Zeppelin-Allee und Bockenheimer Landstraße Hähnchen und trinken dazu Jägermeister; Oskar Negt zieht sich zu einem Mittagsschläfchen zurück; wir schwatzen; Oskar richtet sich in einem Sessel ein, sortiert Pfeife und Zubehör, legt Schallplatte auf; er kommt zur Wohnung herein mit Aktentasche, packt bedächtig aus; die Bücher mit den großen Papierlaschen an den angekreuzten Zitatstellen, sie werden [auf dem gemeinsamen Arbeitstisch] zu Türmen geschichtet. ${ }^{78}$

Aus der Tatsache, dass Negt und Kluge die meiste Zeit ihrer Kooperation nicht in der gleichen Stadt leben, ergibt sich die Notwendigkeit, für die geplante Zusammenarbeit zu reisen, was Diskontinuitäten bedeutet, auch in der Zitation unterschiedlicher Ausgaben, da die Bücher in verschiedenen Bibliotheken entstehen: „Wir leben in zwei verschiedenen Städten. Also haben wir abwechselnd die Bibliothek des einen oder des anderen benutzt. Die Quellen des Buches sind deshalb mehrfach nach verschiedenen Ausgaben zitiert."79

Fühlt sich der Andere am Ort der Schriftproduktion bequem, beginnt das Geschehen am gemeinsamen Arbeitsplatz dann hauptsächlich „klassische Verfahren zur Herstellung formulierter Texte [zu unterlaufen]. Es hält sich länger im Bereich des Rohstoffs, der Abwägung auf." ${ }^{\text {"0 }}$ Abwägung - als juristischer Terminus für die Herbeiführung verhältnismäßiger Entscheidungen - setzt mündliche Kommunikation voraus: Negt berichtet von wochenlangem Sitzen am Tisch, „immer im Blickkontakt. Text für Text diktierend, mit Kopfnicken, Unterbrechungen, Einwürfen den Redefluss des Anderen begleitend.“81

Der Austausch von Wissen aus unterschiedlichen Disziplinen und Erfahrungen beansprucht den Tisch als stabilisierende Kommunikationszone. Mit Niels Werber richten sich die Erwartungen, die Negt und Kluge an das Sitzen am Tisch haben, an ein sozialtechnisches Mittel, das dazu beiträgt, das Problem auf der kommunikativen Ebene so zu formatieren, dass es lösbar wird: ${ }^{82}$ Beide vertrauen darauf, dass es durch das Sitzen am Tisch gelingt, „gemeinsam problematische Fragen nebeneinanderzustellen, sie nacheinander zu analysieren, unmerklich zu einer Einigung zu kommen“. 83 Einerseits sprechen Negt und Kluge von der Notwendigkeit einer „Technik“, eines bewussten Ein- bzw. Kunstgriffs, „damit sich Gegensätze verständigen“. 84

\footnotetext{
78 Ebd., S. 5 .

79 Negt/Kluge, Geschichte und Eigensinn (Anm. 6), S. 1245, Herv. im Orig.

$80 \quad$ Kluge, „Momentaufnahmen“ (Anm. 4), S. 9.

81 Negt, „Meister der Verschlüsselungen“ (Anm. 8), S. 193.

82 Vgl. Niels Werber, „Runde Tische \& eckige Tische. Technologien zur Herstellung von Übereinkunft", in: Hendrik Blumentrath et al. (Hrsg.), Techniken der Übereinkunft. Zur Medialität des Politischen, Berlin: Kadmos 2009, S. 113-131, hier S. 115.

83 Kluge, „Momentaufnahmen“ (Anm. 4), S. 5 .

84 Ebd., S. 7 .
} 
Zugleich betonen sie, dass die Singularität des Arbeits-Verhältnisses in dem Empfangen der Rede des Anderen, dem ausgleichenden, wechselseitigen "Sich-aufeinander-Einlassen“ und in der „Praxis des kommunikativen Urvertrauens" wurzelt, wodurch Konkurrenzmechanismen reduziert werden und die „Ich-Schranke" gesenkt. ${ }^{85}$ In Anspielung auf Schopenhauer verweisen sie schließlich darauf, dass das Vermeiden von "natürliche[r] Rechthaberei“, 86 gegenseitiger Fremdheit und Beunruhigendem ebenso wie das gegenseitige Gewähren von Zeit und Raum den Kern der Zusammenarbeit bilden. ${ }^{87}$

Kooperatives Schreiben erfordert also das Sitzen am gemeinsamen Arbeitstisch und damit eine regulative Kommunikationstechnologie, die das Lustmoment an nicht entfremdeter Schreibarbeit ermöglicht. ${ }^{88}$ An diesem Tisch wären demnach die Selbstregulierungsprozesse der kooperativen Schreibszene zu verorten - wenn das Schreiben von Negt und Kluge nicht auch auf einer Diktierszene basierte, die ebenfalls in den Blick genommen werden muss.

\section{Diktierszene}

Auch die im Raum anwesende Sekretärin ist ein wesentliches Glied in der Kette selbstregulierender Textproduktionsprozesse. Während der Arbeit an Öffentlichkeit und Erfahrung und am ersten Teil von Geschichte und Eigensinn (ca. ab 1978) arbeitete Elfriede Olbrich, Sekretärin bei Adorno bis zu dessen Tod, bei Negt und Kluge. Ihr Name und ihre Funktion bei der Textherstellung werden nachträglich, erstmals in der „Nachbemerkung“ zu Geschichte und Eigensinn, genannt:

Bei der Herstellung des Buches waren wir nicht allein. Uns haben, mit Auswirkung auf den ganzen Inhalt und die Kooperation, Karin Niebergall, Franz Greno und - wie schon bei Öffentlichkeit und Erfahrung - Elfriede Olbrich geholfen. ${ }^{89}$

85 Alexander Kluge und Vincent Pauval, „Einen Robinson gibt es eigentlich nur zu zweit“, in: Langston et al. (Hrsg.), Stichwort (Anm. 3), S. 115-126, hier S. 119.

86 Kluge, „Momentaufnahmen“ (Anm. 4), S. 7. Arthur Schopenhauer, „Eristische Dialektik“, in: ders., Der handschriftliche Nachlaß, Bd. 3: Berliner Manuskripte (1818-1830), hrsg. von Arthur Hübscher, München: dtv 1985, S. 667.

87 Vgl. Kluge, „Momentaufnahmen“ (Anm. 4), S. 7.

88 Richard Langston, „Das ist die umgekehrte Flaschenpost'. Ein montiertes Interview mit Oskar Negt und Alexander Kluge“" in: Richard Langston et al. (Hrsg.), Glass Shards. Echoes of a Message in a Bottle, Göttingen: V\&R unipress 2015, S. 47-75, hier S. 51.

89 Negt/Kluge, Geschichte und Eigensinn (Anm. 6), S. 1245. Ausführlicher zur Rolle der Sekretärin bei Negt und Kluge: Christian Wimplinger, „Sekretärin - Die Frau mit Eigenschaften. Dritte in der Schreib-Kooperation von Negt und Kluge“, in: Christian Schulte et al. (Hrsg.), Plurale Autorschaft, Göttingen: V\&R unipress 2021, S. 167-180. 
Die zugeschriebene „Auswirkung“ der Mitarbeitenden auf den Inhalt der Bücher widerspricht deren bloß randständiger Erwähnung. Dieses Spannungsverhältnis zwischen Autorschaftsinszenierung und geschildertem Produktionsszenario wirft die Frage auf, in welcher Form die Genannten als Kooperationspartner bzw. Kollektiv zu begreifen sind.

In „Momentaufnahmen aus unserer Zusammenarbeit“ bezeichnet Kluge die Mitarbeitenden als „Dritte im Raum“. ${ }^{90}$ Ihre produktive Rolle besteht im Einsammeln, d.h. im Stenographieren der spontan entwickelten Gedanken während des Sprechens und in der Transkription und Bereitstellung der Texte für den nächsten Tag: „Was wir am Vortag getan haben, lesen wir erst, wenn unsere Mitarbeiterin die Texte am nächsten oder übernächsten Tag bringt.“91 In dem Text Adornos Sekretärin (2002) bezeichnet Kluge Elfriede Olbrich als eine „Redakteurin“ und schreibt ihr eine stil-seismographische Funktion zu, wenn es über ihren Gesichtsausdruck während des Stenogramms seines Textes Lernprozesse mit tödlichem Ausgang heißt: „Ob ein Text stimmig ist, sieht man beim Diktat an ihrer Miene. ${ }^{\text {“92 }}$ Darüber hinaus besorgen Elfriede Olbrich und weitere Mitarbeiterinnen Material, recherchieren, bringen sich thematisch, etwa in Form von Buchvorschlägen ein und sorgen, Kluge zufolge, für Widerspruchsfreiheit im anwachsenden Gesamttext. ${ }^{93}$

Die Art und Weise, in der insbesondere Elfriede Olbrich als beteiligtunbeteiligte, namenlose Dritte der Textproduktion gegenwärtig ist, lässt die Paradoxien, Ausschließungsstrategien und damit das Unbewusste des Autorschaftsdiskurses hervortreten. Diejenige, die das Werk schreibt, ohne mit dessen Titel eine Verbindung einzugehen, ist zugleich auch dessen erste Leserin. In dieser zweideutigen Positionierung taucht sie ,zwischen ' den beiden aufeinander bezogenen Autoren in einem Moment auf, den beide als frei von hegemonialem Ansprüchen begreifen - mit Koschorke ein Augenblick in einem offenen und im Hinblick auf totalisierende Systemansprüche skeptischen Gedankenmilieu, der eigentlich auch die Notwendigkeit von

9o Kluge, „Momentaufnahmen“ (Anm. 4), S. 9. Die Formulierung „Dritter“ ist bei Kluge im juristischen Kontext zu verorten, wenn er z.B. im Text meint, dass durch die „erste zupackende Formulierung“ die Sachlage „einem Dritten sofort verständlich zu machen“ (S. 11, Herv. im Orig.) sei. Zum Dritten im Recht, einem Gradmesser für den „Standpunkt eines vernünftigen Dritten“, der als „normativer Modellmensch“ den Blickwinkel eines durchschnittlichen Betrachters repräsentiert, siehe Elena Barnert, „Der Dritte im Recht“, in: Eva Eßlinger et al. (Hrsg.), Die Figur des Dritten. Ein kulturwissenschaftliches Paradigma, Berlin: Suhrkamp 2010, S. 254-263.

91 Kluge, „Momentaufnahmen“ (Anm. 4), S. 9.

92 Alexander Kluge, „Adornos Sekretärin“, in: Ulrich Pohlmann und Matthias Harder (Hrsg.), Stefan Moses. Die Monographie, München: Schirmer/Mosel 2002, S. 144f., hier S. 144.

Nach einer telefonischen Auskunft Alexander Kluges vom 18.o3.2019. 
kulturellen und epistemologischen Verhandlungen im Hinblick auf Autorschaft anzeigen könnte. ${ }^{94}$ Während der "Politischen Universität" in Frankfurt 1968, zu einem Zeitpunkt also, als die Studierenden die Johann Wolfgang Goethe-Universität besetzt hielten, ${ }^{95}$ war Kluge zufolge „eine neue Form von Wissenschaft [...] im Entstehen“ ${ }^{\text {“9 }}$ deren Prämissen in Fluss geraten sind. ${ }^{97}$ Von dieser Verflüssigung bleiben jedoch die Zuschreibungspraktiken von Autorschaft unberührt. Die Buchdeckel und Bibliothekskataloge verschweigen die Mitautorin, auf den Autorenfotos ist sie nicht zu sehen, und doch wird sie nebenbei erwähnt. Trotz der ihr eingeräumten Autonomie und der Betonung ihrer inhaltlichen Bedeutung für den kooperativen Arbeitsprozess sind, wenn es um Autorschaft geht, Hierarchien und Ausschließungsstrategien deutlich sichtbar. Durch diesen Mangel an Aufmerksamkeit auf die textproduzierende Funktion der Dritten im Raum wird die Chance verpasst, jenen „Perspektivwechsel“ zu vollziehen, der im Arbeitsverständnis von Negt und Kluge „[a]m Anfang einer jeden kritischen Arbeit steht“. ${ }^{98}$ Denn so wie die proletarische Öffentlichkeit die bürgerliche kritisiert, kritisiert jedes andere „Liegengebliebene, Ausgegrenzte, in dem noch keine analytische Arbeit drinsteckt, [...] das, was bereits bearbeitet worden ist" ${ }^{\prime}{ }^{99}$ Gleiches gilt auch für die Dritte am Schreibtisch.

Tatsächlich ist die Dritte im Raum in Negt und Kluges Textproduktion an der Schwelle von Mündlichkeit und Schriftlichkeit lokalisiert. Ihre Aufgabe

94 Vgl. Albrecht Koschorke, „Ein neues Paradigma der Kulturwissenschaften“, in: Esslinger et al. (Hrsg.), Die Figur des Dritten (Anm. 9o), S. 9-31. hier S. 13 f.

95 Negt zufolge geht es „dieser Generation um die Integrität des politischen Willens, der die Klagen über den Verwaltungsmassenmord des Dritten Reiches nicht ertragen kann, ohne sie in Aktionen gegen jede Form des gegenwärtigen Mordes umzusetzen“ (Oskar Negt, „Studentischer Protest - Liberalismus - ,Linksfaschismus“', in: Kursbuch 13 (1968), S. 179189, hier S. 184). Gleichzeitig sind direkte Aktionen, also Blockaden, Besetzungen, Proteste etc. selbst der Gefahr ausgesetzt, blind und zerstörerisch zu verlaufen: Eine Skepsis gegenüber der direkten Aktion, die sich in Habermas' - später revidiertem - Vorwurf des Linksfaschismus andeutet, ist aber auch am Arbeitstisch zu beobachten, wenn es etwa um die Verschriftlichung eines mündlich formulierten Gedankens geht. Kluge behauptet, „daß derjenige, der etwas abbildet und formuliert, gerade nicht kritisch prüft, dies tut in dem Moment der andere“ (Kluge, „Momentaufnahmen“ [Anm. 4], S. 9). Durch die Mitarbeit eines Dritten während des Schreibprozesses kommt Umsicht und Verantwortung in das notwendig blinde Handeln. Die primäre Funktion der Sekretärin ist aber nicht, die Inhalte kritisch zu prüfen, sondern die Momente in schriftlicher Form aufzunehmen. Ohne diese lebenssituativ verankerte Fixierung wären sie, so Kluge, verloren.

96 Kluge, „Momentaufnahmen“ (Anm. 4), S. 12.

97 Vgl. ebd., S. 9 .

$98 \quad$ Negt/Kluge, Geschichte und Eigensinn (Anm. 6), S. 87.

99 Ebd. 
besteht im Transfer einer mündlich artikulierten Einsicht, die man "gleich diktieren [muss] “, 100 in eine schriftliche Form für die weitere Textbearbeitung. Darüber hinaus agiert die Dritte aber auch auf der Schwelle zwischen Diskurs und Narration, insofern sie als Zuhörerin von Erzählungen adressierbar ist. In dieser Figur müsste sich eigentlich die Forderung der antiautoritären Bewegung kristallisieren, wonach theoretische Inhalte stets auf die eigene Lebenssituation rückbezogen und narrativ eingeholt werden müssen. De facto kommt aber der Lebens- und Arbeitsbereich der angestellten Sekretärin Elfriede Olbrich wie der aller anderen Angestellten in den ersten beiden Büchern Negt und Kluges an keiner Stelle vor. In dem Text Adornos Sekretärin, den Kluge zu einer Bildmonographie von Stefan Moses 2002 beisteuert und die auch das hier abgedruckte Foto Elfriede Olbrichs enthält (Abb. 7.2), geht es wesentlich um den Rahmen, in dem das Bild entstanden ist, um die Selbstinszenierung Elfriede Olbrichs und um die Funktion von „Madmoiselle Olbrich ${ }^{4101}$ in Adornos Textproduktion.

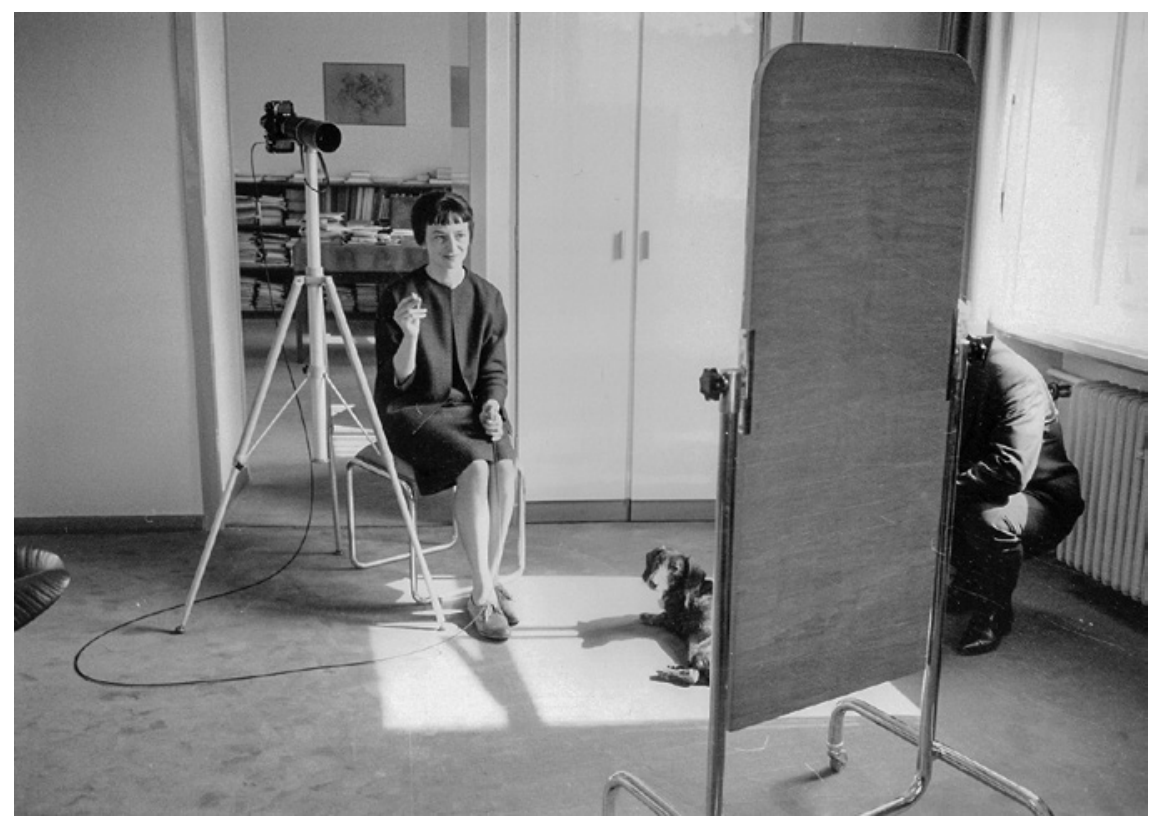

Abb. 7.2 Elfriede Olbrich, Selbstporträt mit Spiegel. Fotografie: stefan moses | \ stefan moses archiv

100 Kluge, „Momentaufnahmen“ (Anm. 4), S. 11.

101 Kluge, „Adornos Sekretärin“ (Anm. 92), S. 144. 
Michael Ott, der die Diktierszene im Rahmen der Schreibszenenforschung diskutiert, ${ }^{102}$ stellt fest, dass das Diktat Machtstrukturen zwischen Diktierenden und Schreibenden verschleiert, und dass es die Frage der Autonomie oder Heteronomie von Autorschaft veruneindeutigt, indem die Autorschaft vom Manuskript getrennt wird. Das trifft auch auf die Diktierszene von Negt und Kluge zu. Beider Kooperation gründet in dem von Kluge so benannten „Prinzip Mündlichkeit“, das auf die spontane Artikulation und Produktion von Gedanken in Anwesenheit eines anderen setzt. ${ }^{103}$ Dabei spielt die je eigene Art des Sprechens eine zentrale Rolle, wenn, wie bei Kleist, ${ }^{104}$ Silben gedehnt werden, um Zeit zu gewinnen, oder, wie bei Negt, rhythmisches PfeifeRauchen die Art des Sprechens strukturiert. ${ }^{105}$ Im Anschluss an Benjamin versteht Kluge mündliches Erzählen als Verarbeitung von Erfahrungen, die, weil immer neue Gedanken und Formulierungen als „Rohstoff" ${ }^{106}$ heranströmen, aufgrund ihrer Flüchtigkeit unmittelbar diktiert werden müssen. ${ }^{107}$ Erst unter Einbeziehung des Diktats in das Ensemble der Textproduktion kann also von einem kollektiven Schreiben gesprochen werden, in dem die Sekretärin eine entscheidende Rolle innehat. Denn Negt und Kluges „orientierende[] Theorie“ hält stets einen Praxisbezug aufrecht, der nach „Aushilfen“ und nach Auswegen aus kritischen Situationen sucht. ${ }^{108}$ Diese Suche der schreibenden Philosophen ist "auf den glücklichen Moment [angewiesen]“, 109 der einen Eingriff in die offene Situation erlaubt. ${ }^{110}$ Konzentriert sich die Aufmerksamkeit derart auf das Einsammeln solch glücklicher Momente, stellt sich die Frage nach einem geeigneten Aufzeichnungsmedium. Negt und Kluge sprechen in diesem Zusammenhang auch von einem „innere[n] Seismograph[en]“, ${ }^{111}$ den

102 Michael Ott, „,Setz dich. Schreib. Diktier-Szenen bei Schiller und Kleist“, in: Claudia Lubkoll und Claudia Öhlschläger (Hrsg.), Schreibszenen: Kulturpraxis - Poetologie Theatralität, Freiburg/Br., Berlin, Wien: Rombach 2015, S. 191-213.

103 Kluge, „Momentaufnahmen“ (Anm. 4), S. 1of.

104 Kluge und Negt beziehen sich häufig auf Kleists Über die allmähliche Verfertigung der Gedanken beim Reden, z.B. in Kluge, „Momentaufnahmen“ (Anm. 4), S. 1 of.

105 Vgl. ebd., S. 11.

106 Walter Benjamin, „Der Erzähler. Betrachtungen zum Werk Nikolai Lesskows“, in: ders., Gesammelte Schriften, Bd. 2.1, hrsg. von Rolf Tiedemann und Hermann Schweppenhäuser, Frankfurt/Main: Suhrkamp 1991, S. 438-465, hier S. 464.

107 Kluge, „Momentaufnahmen“ (Anm. 4), S. 11.

108 Negt/Kluge, Geschichte und Eigensinn (Anm. 6), S. 84.

109 Ebd.

110 Das Bild der schreibenden ArbeiterInnen der Solicarność-Bewegung am Anfang von Negt/Kluge, Geschichte und Eigensinn (Anm. 6), S. 18f., oder die Geschichte über Rosa Luxemburg und die fusionierende Gruppe zeigen, dass für ein Kollektiv kollektiv geschrieben wird.

111 Negt/Kluge, Geschichte und Eigensinn (Anm. 6), S. 84. 
unter anderem die Sekretärin verkörpert. Ihre Miene indiziert nicht nur die Stimmigkeit des Textes, sondern auch den rechten Augenblick, in dem Greifbares und Festhaltenswertes auftaucht: Die auf „unsere Rede- und Diktierweise eingestellte“ Sekretärin nimmt, so Negt, „nicht nur die ausdrücklichen Diktate“ auf, „sondern auch Diskussionen zwischen uns, wenn sie den Eindruck gewinnt, dass wir diese im formalisierten Text gebrauchen könnten.“112

\section{Kooperation, Kollektiv?}

Wenn in den Texten, in denen Negt und Kluge ihre Arbeitsweise reflektieren, von Kooperation, gemeinsamer Produktion, Zusammenarbeit und mündlicher Kommunikation die Rede ist, wird Kooperation und Kollektiv folgendermaßen gefasst: Das Wort „kollektiv“ bezieht sich zum einen auf die Gesellschaft, ihre Geschichte, Produkte und Erfahrungen, und zum anderen auf die fusionierende Gruppe und ihr Sammeln und Auflesen. Kollektives Schreiben verstehen Negt und Kluge als Vorgang der gemeinsamen Kollektions- und Konstellationsbildung von lebensbezogenen Erfahrungen, durch deren permanente Permutation Auswege aus kritischen Situationen für einen bestimmten Moment erkennbar werden.

Um von kollektivem Schreiben und Schreib-Kollektiven im Sinne der Fragestellung dieses Bandes sprechen zu können, muss über Negt und Kluges Perspektivierung gemeinsamen Schreibens hinaus der Umgang mit Dingen und mit Menschen - dem Tisch und der im Raum mitanwesenden Schreibkraft als weiteren Akteuren im Schreibszenario in den Blick genommen werden. Sprechen Negt und Kluge von Autorschaft im Sinne von Textverantwortlichkeit, geht es alleine um die beiden, die während der gemeinsamen Arbeit aber ausschließlich mündlich agieren. Das betrifft die textkonstitutive Arbeit im Sinne von gemeinsam am Tisch sitzen, sprechen, blättern, Notizen machen, Zettel beschriften. Die Verschriftlichung aber ist Aufgabe der Mitarbeiterin.

Dass der Schreibtisch nicht explizit als Akteur in die Schriftproduktion einbezogen ist, dürfte seine historische Begründung auch darin haben, dass diese Figur und die mit ihr verbundene Täterschaft im aktiven Gedächtnis der Nachkriegszeit seit den 196oer Jahren negativ, als Ort bürokratischer Fernsteuerungen besetzt ist, wie Markus Krajewski ausgeführt hat ${ }^{113}$ und wie auch

\footnotetext{
112 Negt, „Über Vertrauen und Kooperation“ (Anm. 14), S. 18.

113 Vgl. Markus Krajewski, „Am Grünen Tisch. Skizze zu einer kurzen Geschichte bürokratischer Fernsteuerungen“, in: Dirk van Laak und Dirk Rose (Hrsg.), Schreibtischtäter: Begriff - Geschichte - Typologie, Göttingen: Wallstein 2018, S. 95-112.
} 
Negts Rede vom „Verwaltungsmassenmord“ zeigt. ${ }^{114}$ Negt und Kluges mündliches, nicht-schreibendes Setting steht in Opposition zu der Täterschaft dieser Figur, deren Tat ja gerade das Schreiben ist. Auch Kluges These, dass nur im vertrauensbasierten Gespräch sichergestellt werden kann, dass „verantwortlich [ge]handel[t]" wird,115 muss als weiterer Grund für die deutliche Markierung der Abwesenheit des Schreibens am gemeinsamen Tisch in Betracht gezogen werden.

Der Glaube an die Gestaltbarkeit von Geschichte ist nach Ingrid GilcherHoltey mit dem Scheitern der Protestbewegung ins Wanken geraten, was den Beginn der Postmoderne markiert. ${ }^{116}$ Das Ende der Moderne berührt aber nicht nur die Geschichtskonzeption, sondern auch die Vorstellung des Menschen und seine Handlungsmöglichkeiten im Kollektiv, in das mehr und mehr auch nicht-menschliche Akteure einbezogen werden. Dass Negt und Kluge solch einen "verengten Modernitätsbegriff“ nicht mittragen, ${ }^{117}$ zeigt alleine schon der 2001 gewählte Titel ihrer "gemeinsame[n] Philosophie“ Der unterschätzte Mensch, der einem frühen Vorschlag Negts zufolge noch „Der überschätzte Mensch" lauten sollte. ${ }^{118}$ Das Bestehen auf der Kategorie ,Mensch' und die Kontinuität in der Suche nach seinen „verdeckte[n], verschüttete[n], verdrehte[n] Eigenschaften “119 rebellieren gegen die Vermenschlichung von Dingen und die Verdinglichung von Menschen, wie sie für einen postmodernen Kollektivbegriff exemplarisch sind.

114 Negt, „Studentischer Protest“ (Anm. 94), S. 184.

115 Kluge, „Momentaufnahmen“ (Anm. 4), S. 9.

116 Ingrid Gilcher-Holtey, 1968. Eine Zeitreise, Frankfurt/Main: Suhrkamp 2008, S. 199 f.

117 Negt/Stollmann/Schulte, „Der Maulwurf“ (Anm. 2), S. 13.

118 Ebd., S. 11, Herv. im Orig.

119 Ebd., S. 12. 